\title{
Improved Angina Symptoms Following Coronary Sinus Flow Reducer Implantation in a Patient with Refractory Angina and Chronic Total Occlusion: A Case Report
}

\author{
Crt Langel', Mrak Miha², Nejc Pavsic ${ }^{2}$, Matjaz Bunc ${ }^{2 *}$ \\ ${ }^{1}$ Clinical Institute of Radiology, University Medical Center Ljubljana, Zaloska, Ljubljana, Slovenia \\ ${ }^{2}$ Clinical Department of Cardiology, University Medical Center Ljubljana, Zaloska, Ljubljana, Slovenia \\ Email: *mbuncek@yahoo.com
}

How to cite this paper: Langel, C., Miha, M., Pavsic, N. and Bunc, M. (2019) Improved Angina Symptoms Following Coronary Sinus Flow Reducer Implantation in a Patient with Refractory Angina and Chronic Total Occlusion: A Case Report. International Journal of Clinical Medicine, 10, 604-612.

https://doi.org/10.4236/ijcm.2019.1011049

Received: July 16, 2019

Accepted: November 11, 2019

Published: November 14, 2019

Copyright $\odot 2019$ by author(s) and Scientific Research Publishing Inc. This work is licensed under the Creative Commons Attribution International License (CC BY 4.0).

http://creativecommons.org/licenses/by/4.0/

\begin{abstract}
Background: Due to the aging population and increased survival of the patients with coronary artery disease, there is an increasing number of patients with debilitating angina refractory to optimal medical treatment who are not candidates for revascularization. In case of low ischemic load, the treatment of stable refractory angina is aimed at symptom reduction. There are several new treatment methods targeting myocardial ischemia available, including coronary sinus flow reducer (CFR) implantation. Case Report: We report a case of a patient suffering from CCS class IV angina despite optimal medical therapy, with further revascualrization options exhausted, who was successfully treated with coronary sinus flow reducer (CFR). Besides technical skill to reach ostium of coronary sinus, the most important technical tip is precise positioning of the CFR. The reduction of angina symptoms started after epithelisation of CFR frame, usually 6 - 7 weeks after implantation. At 6-month follow-up, the patient reported a marked reduction of angina symptoms, with CCS grade improving by three classes (from IV to I). At 10-month follow-up, the sustainment of CCS grade I angina symptoms was reported by the patient. Conclusions: We conclude that CFR can be safely and successfully implanted in patients suffering from refractory angina. Considerable improvements in CCS grade may be experienced in certain cases.
\end{abstract}

\section{Keywords}

Chest Pain, Refractory Angina Pectoris, Myocardial Ischemia, Coronary Sinus Flow Reducer, Chronic Total Occlusion 


\section{Introduction}

Advancements in drug and device therapy, along with the aging population, have increased the life expectancy of the patients with coronary artery disease (CAD) [1]. This has also resulted in the increased prevalence of those CAD patients who have chronic angina pectoris refractory to medical treatment and who are ineligible for further surgical or percutaneous revascularization [2] [3]. Patients with reversible ischemia-related angina lasting $\geq 3$ months despite optimal medical therapy and revascularization options exhausted are said to have refractory angina (RA). This group of patients comprises an estimated 5\%-10\% of all diagnosed angina cases. They often use several anti-ichaemic drugs, experience a poor quality of life due to deleterious symptoms and are frequently hospitalized [3].

Coronary sinus flow reducer (CFR) stent is an implantable device aimed at reducing angina pectoris symptoms by decreasing the cross-section of the coronary sinus (CS) and increasing venous back pressure. This in effect redirects blood flow to subendocardium, increases collateral blood flow and, presumably, induces neoangiogenesis [4] [5]. Combined, these mechanisms increase perfusion of the more ischemic regions of the myocardium, resulting in alleviation of angina symptoms, improved myocardial contraction and reduced left ventricular end-diastolic pressure [6].

CFR is a stainless steel balloon-expandable hourglass-shaped stent inserted into the CS via transjugular approach. It is a percutaneous analogue of surgical partial ligation of the CS that was first performed by Beck and Leighninger in 1954 and was-despite significantly improving angina symptoms and reducing 5 -year mortality rate-later discontinued in part due to widespread acceptance of the coronary artery bypass graft (CABG) surgery [7].

Studies of CFR so far have demonstrated the device and its implantation procedure to be feasible, safe and efficacious. The COronary SInus Reducer for treatment of Angina (COSIRA) multicentre, prospective, double-blind, shamcontrolled study showed significantly higher percentage of patients with Canadian Cardiovasular Society (CCS) classification improvement of at least one functional class in the treatment group in comparison to the sham-controlled group ( $71 \%$ and $42 \%$, respectively) [8]. Two smaller studies also demonstrated significant improvements in objective myocardial ischemia measures, such as exercise time or the mean change in wall-motion index assessed by dobutamine echocardiography [9] [10]. Our heart centre has been employing CFR in RA treatment since 2016 with overall results so far closely matching the COSIRA trial outcomes.

In this case report, we describe a patient with multiple coronary diseases, after CABG and unsuccessful PCI of CTO RCA. He was suffering from CCS class IV angina with multiple comorbidities, including diabetes and aortic valve replacement. After CFR implantation he showed a sustained improvement of angina symptoms at 6- and 10-month follow-up, demonstrating the safety and 
efficacy of CFR implantation in such patients.

\section{Case Report}

80-year-old man with a history of hypertension, dyslipidemia, type 2 diabetes treated with oral antihyperglycemic therapy, benign prostatic hyperplasia, peripheral arterial disease, atrial fibrillation, non-ST-elevation myocardial infarction, CABG using left internal mammary artery (LIMA) grafted to left anterior descending coronary artery (LAD) and venous graft grafted to obtuse marginal 1 (OM 1), and aortic valve replacement using bioprosthetic valve presented to our clinic with CCS class IV angina despite optimal medical therapy (acetylsalicylic acid $100 \mathrm{mg}$, nebivolol $5 \mathrm{mg}$ twice daily, ramipril $5 \mathrm{mg}$ twice daily).

Cardiac echography showed impaired left ventricular systolic function with EF of $48 \%-50 \%$, with basal, inferior, inferior septal and inferior lateral wall motion abnormalities. Aortic bioprosthetic valve showed normal function.

Coronary angiography showed 2-vessel coronary artery disease with RCA chronic total occlusion (CTO) with left to right Rantop 2 collateral flow and an important stenosis of proximal LAD. Surgical revascularization was our first option. After patient refused surgery we performed proximal LAD stenting with DES (Synergy $3.0 \times 12 \mathrm{~mm}$ ). PCI CTO with different approaches (antegrade, retrograde) was not successful. Finally, dissection of the mid RCA occurred during PCI attempts (Figure 1). Due to RA persisting 3 months after PCI despite optimal medical therapy (bisoprolol $5 \mathrm{mg}$, perindopril $10 \mathrm{mg}$, ranolazine $2 \times 500$ $\mathrm{mg}$, rosuvastatine $30 \mathrm{mg}$ ), a multi-disciplinary heart team decided that CFR implantation was the optimal treatment approach for this patient.

Ultrasound-guided right internal jugular vein puncture was performed, followed by multipurpose catheter insertion and coronary sinus ostium cannulation using fluoroscopic guidance. A 9 French guiding catheter with the CFR stent (Neovasc Inc., Richmond B.C., Canada) was placed inside the coronary sinus at the appropriate insertion point, i.e. at least $2 \mathrm{~cm}$ distal to the ostium to exclude small cardiac veins draining the right coronary artery (RCA) venous return, all the while excluding the larger, more distal side branches draining the left coronary artery (LCA) venous return. The catheter-mounted hourglass-shaped balloon was inflated at 5 atm for 30 seconds, expanding the reducer stent into its functional shape (Figure 2). The recommended $10 \%-20 \%$ device oversizing relative to the coronary sinus cross-section was achieved in order to ensure proper anchoring, prevent device migration and induce endothelization of the stent's mesh structure. After balloon deflation and the removal of the catheter, venography showed successful reducer stent position and anchoring. Complications such as stent occlusion, coronary sinus dissection or perforation, or bleeding at the jugular puncture site were excluded. The patient received dual antiplatelet therapy comprising acetylsalicylic acid and clopidogrel for the duration of 6 months.

The reduction of angina symptoms began $6-7$ weeks after implantation. At 6-month follow-up, the patient showed marked reduction of angina symptoms, 

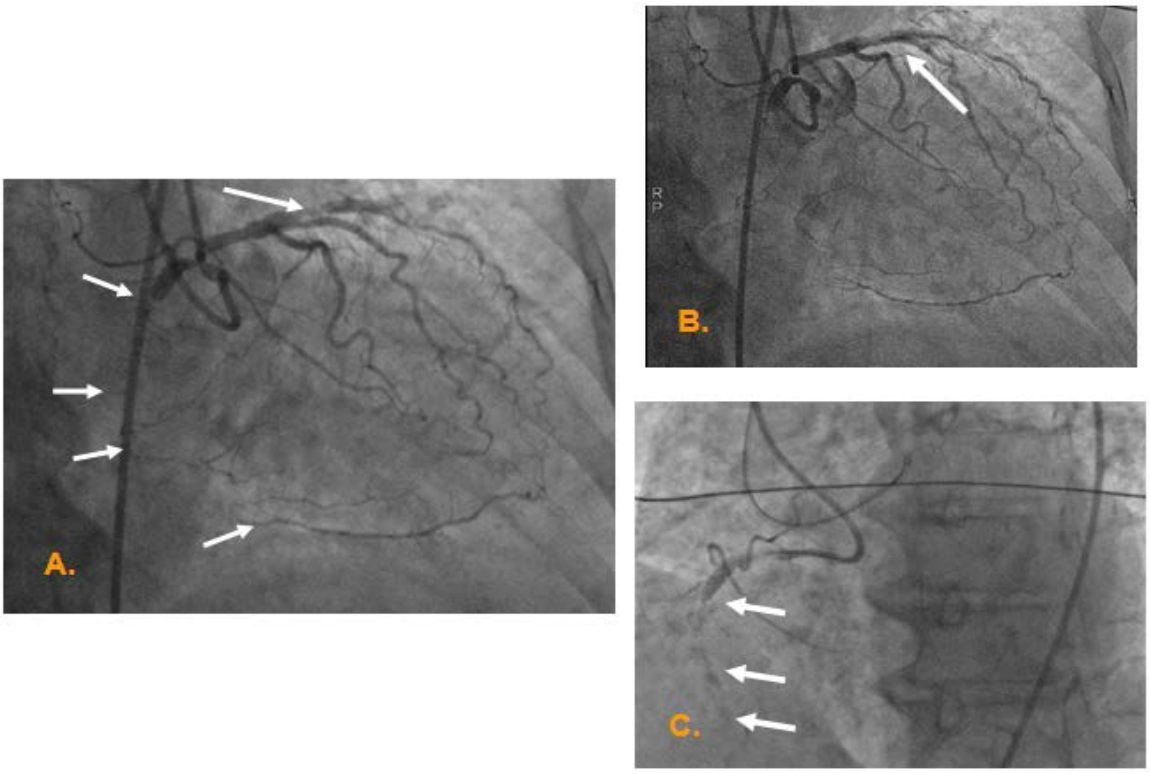

Figure 1. Coronary angiography. (A) Biradial injection of LM and RCA. Proximal occlusion of RCA. Severe stenosis of proximal LAD; (B) LAD after DES stenting; (C) Unsuccessful PCI of CTO RCA: combined tehnique (antegrade/retrograde). Note the disection of RCA.
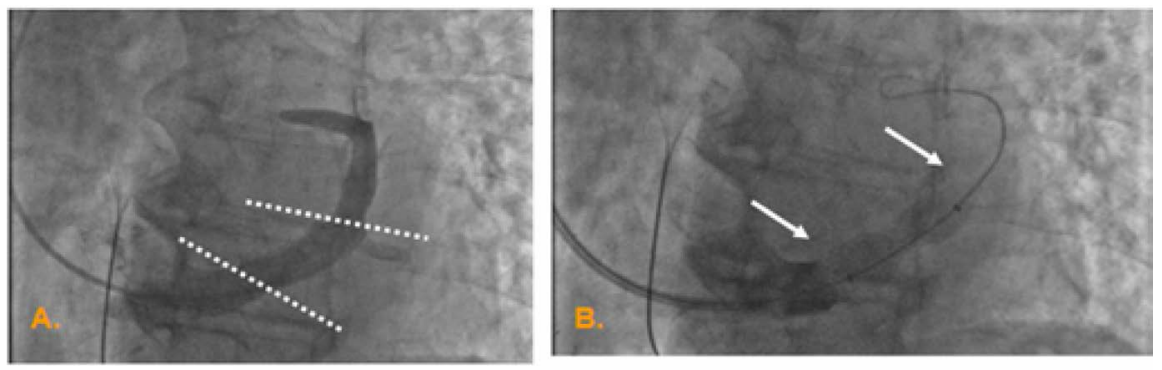

LAO $30^{\circ}$

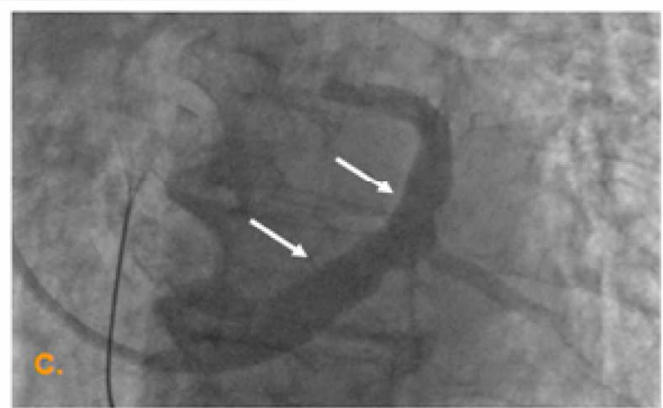

Figure 2. Coronary flow reducer (CFR) implantation. LAO $30^{\circ}$. (A) Coronary sinus angiography. Right jugular approach. Left amplatz 1 shaped catether. Anatomic target position for CFR implantation; (B) Coronary sinus angiography during CFR-balloon inflation. Note the supportive wire (Amplatz extrasupport). No parallel flow to expanded CFR (5 atm continuous pressure delivered through indeflator); (C) Final position of the CFR after successful implantation.

with CCS grade improving by three classes (from IV to I). At 10-month follow-up, the sustainment of CCS grade I angina symptoms was reported by the patient. 


\section{Discussion}

Patients with CAD have increased life expectancy, to some extent due to advancements in drug and device therapy and in part due to the aging population. In general, we are faced with an increased number of patients with RA. Despite having a long-term mortality of under $4 \%$ per year, approaching that of the patients with chronic coronary syndrome (1.5\% per year, $6.1 \%$ in 5 years for patients with no prior MI, $10.8 \%$ in 5 years for patients with prior MI), the incapacitating nature of angina has a significant negative effect on quality of life in patients with RA [3] [10] [11]. Up to $30 \%$ of stable CAD still experience angina symptoms 1 year after revascularization [12]. Data derived from cardiac cath-lab registries showed that $6 \%-12 \%$ of the patients referred to angiography with evidence of ischemia were ineligible for traditional revascularization [13].

Several methods have been under investigation to mitigate the symptoms of RA. First-line treatment options include beta-blockers, ivabradine, calcium channel blockers, nitrates, ATP-sensitive potassium channel openers, late sodium current inhibitors and rho-kinase inhibitors [14]. According to Diamond recommendations and novel ESC guidelines on chronic coronary syndrome, patients should be treated with a combination of event prevention and angina relief medical therapy [15] [16]. Approximately $10 \%$ of RA patients receiving one or more of the medical treatment choices remain symptomatic [17].

There are some additional treatment options of RA, including external enhanced counterpulsation, extracorporeal shockwave therapy, spinal cord stimulation, internal mammary artery implants, transmyocardial laser revascularisation, cell therapy and gene therapy. None of these approaches, however, have yet become widely used [18].

Lately, the CFR implantation has shown promising results in alleviating angina symptoms and improving quality of life. $70 \%-80 \%$ of patients experience improvement in CCS class, while according to the COSIRA data there is also up to $30 \%$ of placebo effect in angina symptoms' improvement [8]. Since RA treatment is aimed at symptom reduction, its goal is achieved even in the placebo population. According to ESC guidelines, CFR is included as a B evidence level, IIb recommendation class RA treatment option [16]. Available clinical data suggest CFR should be indicated in RA patients with proven ischemia in LAD and LCX territory. Positive effects of CFR implantation were also proven in microvascular angina [19]. In a single case with RA following successful CABG, CFR implantation was followed by complete disappearance of nocturnal events, significant improvement in exercise tolerance and angina improvement by two CCS classes (IV to II) at 3-month follow-up were reported [14]. We found no other published data on angina improvement following CFR implantation in CTO RCA patients.

In spite of regular selection of patients, we may expect a non-response cohort of patients up to $20 \%-30 \%$. Some possible mechanisms may be: ischemia arising from the territory of RCA, symptoms caused by heart failure rather than ischemia, non-ischemic chest pain, the presence of an alternative drainage ven- 
ous system of the myocardium into the right ventricle (the Thebesian venous system), and incomplete endothelization of the stent with inadequate pressure gradient across the device [20]. Some of the proposed mechanisms need further clinical evaluation. It has been shown that peri-procedural measurement of the differential pressure between right atrial pressure before implantation and coronary sinus systolic pressure during balloon occlusion might be able to identify the non-responder population; the patients who achieve a high peri-procedural differential pressure are more prone to have a high post-implantation, post-endothelization pressure gradient across the device and thus a higher probability of good response regarding angina symptoms [21].

CFR implantation is a safe procedure, with only one coronary sinus perforation reported so far [22]. Other potential complications include migration of the device, thrombotic occlusion of the stent's lumen and dissection of the coronary sinus. In our case report, we present a successful CFR implantation with good clinical reduction of angina. At this point, we cannot prove the mechanism of the improvement. Nevertheless, the clinical dynamic ensued according to previous clinical data, i.e. the improvement of angina symptoms appeared 6 - 7 weeks after CFR implantation. This is the expected time period necessary for CFR mesh endothelization, which is the critical point for hemodynamic effects resulting in venous backpressure build-up to take place.

So far we have experience with 5 CFR implantations in CTO RCA patients. Reduction of RA in this population seems to be lower than in the overall CFR population, $60 \%$ vs. $75 \%$, respectively. We may speculate that CFR might not be as effective in the cases of large ischemic area. Additional clinical data are required to support our hypothesis.

There is an ongoing discussion concerning the possibility of using CFR in patients with chronic angina who are candidates for percutaneous revascularization of CTOs. There are data from our CTO registry confirming the clinical efficacy of successful PCI of LAD, RCA, LCX in reducing RA in 6-year follow-up (55\%, 35\%, less than 5\%, respectively) [23]. However, in the light of the recent CTO studies' conflicting results, there is still a debate regarding the clinical efficacy of CTO interventions at improving angina frequency, physical limitation and quality of life [24] [25]. In addition, CTO revascularizations do not offer prognostic benefits but rather aim at improving angina symptoms, which is exactly what the Reducer has already proven to achieve. Furthermore, while CTO revascularization is technically challenging and often time-consuming, CFR implantation is a relatively simpler and usually shorter ( 30 - 45 minutes) procedure [26]. Additional clinical data are necessary to define ischemic load burden that might be addressed by either CFR implantation or CTO PCI, both aiming at reduction of angina symptoms.

\section{Conclusion}

Optimal medical therapy is the initial treatment approach to stable CAD. In case 
of proven ischemia exceeding $10 \%$ of the myocardium, revascularization options should be considered [27]. However, patients with angina refractory to optimal medical treatment and with revascularization options exhausted make up a substantial portion of all patients with CAD and their number is expected to increase in the years to come. CFR implantation has proved to be an effective and safe RA treatment method. Both wider acceptance of this novel technique into regular clinical practice and broadening of the patient population (e.g. including patients with RA due to CTO) are expected in the near future. We have shown a case of a patient with remarkable CCS class improvement of angina symptoms, demonstrating the potential of CFR implantation to have a transformative impact on the quality of life of patients with RA. Further clinical studies are required to evaluate CFR implantation as a complementary treatment strategy in addition to optimal medical therapy and/or revascularization.

\section{Conflicts of Interest}

The authors declare no conflicts of interest regarding the publication of this paper.

\section{References}

[1] Banai, S., Ben Muvhar, S., Parikh, K.H., et al. (2007) Coronary Sinus Reducer Stent for the Treatment of Chronic Refractory Angina Pectoris: A Prospective, Open-Label, Multicenter, Safety Feasibility First-in-Man Study. Journal of the American College of Cardiology, 49, 1783-1789. https://doi.org/10.1016/j.jacc.2007.01.061

[2] Mannheimer, C., Camici, P., Chester, M.R., et al. (2002) The Problem of Chronic Refractory Angina; Report from the ESC Joint Study Group on the Treatment of Refractory Angina. European Heart Journal, 23, 355-370. https://doi.org/10.1053/euhj.2001.2706

[3] Williams, B., Menon, M., Satran, D., et al. (2010) Patients with Coronary Artery Disease Not Amenable to Traditional Revascularization: Prevalence and 3-Year Mortality. Catheterization and Cardiovascular Interventions, 75, 886-891. https://doi.org/10.1002/ccd.22431

[4] Giannini, F., Aurelio, A., Jabbour, R.J., et al. (2017) The Coronary Sinus Reducer: Clinical Evidence and Technical Aspects. Expert Review of Cardiovascular Therapy, 15, 47-58. https://doi.org/10.1080/14779072.2017.1270755

[5] Weigel, G., Kajgana, I., Bergmeister, H., et al. (2007) Beck and Back: A Paradigm Change in Coronary Sinus Interventions-Pulsatile Stretch on Intact Coronary Venous Endothelium. The Journal of Thoracic and Cardiovascular Surgery, 133, 1581-1587. https://doi.org/10.1016/j.jtcvs.2006.12.044

[6] Konigstein, M., Verheye, S., Jolicœur, E.M. and Banai, S. (2016) Narrowing of the Coronary Sinus: A Device-Based Therapy for Persistent Angina Pectoris. Cardiology in Review, 24, 238-243. https://doi.org/10.1097/CRD.0000000000000101

[7] Beck, C.S. and Leighninger, D.S. (1954) Operations for Coronary Artery Disease. The Journal of the American Medical Association, 156, 1226-1233. https://doi.org/10.1001/jama.1954.02950130006002

[8] Verheye, S., Jolicœur, E.M., Pettersson, T., et al. (2015) Efficacy of a Device to Narrow the Coronary Sinus in Refractory Angina. The New England Journal of Medi- 
cine, 372, 519-527. https://doi.org/10.1056/NEJMoa1402556

[9] Konigstein, M., Meyten, N., Verheye, S., et al. (2014) Transcatheter Treatment for Refractory Angina with the Coronary Sinus Reducer. EuroIntervention, 9, 1158-1164. https://doi.org/10.4244/EIJV9I10A196

[10] Henry, T.D., Satran, D., Hodges, J.S., et al. (2013) Long-Term Survival in Patients with Refractory Angina. European Heart Journal, 34, 2683-2638. https://doi.org/10.1093/eurheartj/eht165

[11] Sorbets, E., Fox, K.M., Elbez, Y., et al. (2019) Long-Term Outcomes of Chronic Coronary Syndrome Worldwide: Insights from the International CLARIFY Registry. European Heart Journal, ehz660. https://doi.org/10.1093/eurheartj/ehz660

[12] Abdallah, M.S., Wang, K., Magnuson, E.A., et al. (2017) Quality of Life after Surgery or DES in Patients with 3-Vessel or Left Main Disease. Journal of the American College of Cardiology, 69, 2039-2050. https://doi.org/10.1016/j.jacc.2017.02.031

[13] Bernstein, S., Brorsson, B., Aberg, T., et al. (1999) Appropriateness of Referral of Coronary Angiography Patients in Sweden. Heart, 81, 470-477.

https://doi.org/10.1136/hrt.81.5.470

[14] Grandjean, T., Haefliger, D., Arroyo, D. and Cook, S. (2018) Coronary Sinus Reduction for the Treatment of Refractory Angina: A Novel Tool for the Treatment of Angina Pectoris Resistant to Optimal Conventional Therapy. Cardiovascular Medicine, 21, 170-173. https://doi.org/10.4414/cvm.2018.00558

[15] Ferrari, R., Camici, P.G., Crea, F., et al. (2018) Expert Consensus Document: A 'Diamond' Approach to Personalized Treatment of Angina. Nature Reviews Cardiology, 15, 120-132. https://doi.org/10.1038/nrcardio.2017.131

[16] Knuuti, J., Wijns, W., Saraste, A., et al. (2019) 2019 ESC Guidelines for the Diagnosis and Management of Chronic Coronary Syndromes: The Task Force for the Diagnosis and Management of Chronic Coronary Syndromes of the European Society of Cardiology (ESC). European Heart Journal, ehz425.

[17] McGillion, M., Arthur, H.M., Cook, A., et al. (2012) Management of Patients with Refractory Angina: Canadian Cardiovascular Society/Canadian Pain Society Joint Guidelines. Canadian Journal of Cardiology, 28, S20-S41. https://doi.org/10.1016/j.cjca.2011.07.007

[18] Abawi, M., Nijhoff, F., Stella, P.R., et al. (2016) Safety and Efficacy of a Device to Narrow the Coronary Sinus for the Treatment of Refractory Angina: A Single-Centre Real-World Experience. Netherlands Heart Journal, 24, 544-551. https://doi.org/10.1007/s12471-016-0862-2

[19] Giannini, F., Baldetti, L., Ielasi, A., et al. (2017) First Experience with the Coronary Sinus Reducer System for the Management of Refractory Angina in Patients without Obstructive Coronary Artery Disease. JACC: Cardiovascular Interventions, 10, 1901-1903. https://doi.org/10.1016/j.jcin.2017.06.062

[20] Konigstein, M., Giannini, F. and Banai, S. (2018) The Reducer Device in Patients with Angina Pectoris: Mechanisms, Indications, and Perspectives. European Heart Journal, 39, 925-933. https://doi.org/10.1093/eurheartj/ehx486

[21] Baldetti, L., Colombo, A., Banai, S., et al. (2018) Coronary Sinus Reducer NonResponders: Insights and Perspectives. EuroIntervention, 13, 1667-1669. https://doi.org/10.4244/EIJ-D-17-00626

[22] Cortese, B., Di Palma, G. and Latini, R. (2018) Coronary Sinus Perforation during Reducer Implantation. Catheterization and Cardiovascular Interventions, 91, 1291 1293. https://doi.org/10.1002/ccd.27549

[23] Kranjec, I., Zavrl Dzananovic, D., Mrak, M. and Bunc, M. (2019) Robustness of 
Percutaneously Completed Coronary Revascularization in Stable Coronary Artery Disease: Obstructive versus Occlusive Lesions. Angiology, 70, 78-86. https://doi.org/10.1177/0003319718767737

[24] Park, S.-J. (2017) Drug-Eluting Stent versus Optimal Medical Therapy in Patients with Coronary Chronic Total Occlusion: DECISION CTO Randomized Trial. American College of Cardiology Annual Scientific Session 2017, Washington, DC, USA, 18 March 2017.

[25] Werner, G.S., Martin-Yuste, V., Hildick-Smith, D., et al. (2018) A Randomized Multicentre Trial to Evaluate the Utilization of Revascularization or Optimal medical Therapy for the Treatment of Chronic Total Coronary Occlusions. European Heart Journal, 39, 2484-2493. https://doi.org/10.1093/eurheartj/ehy220

[26] Cheng, K. and de Silva, R. (2018) New Advances in the Management of Refractory Angina Pectoris. European Cardiology Review, 13, 70-79. https://doi.org/10.15420/ecr.2018:1:2

[27] Neumann, F.J., Sousa-Uva, M., Ahlsson, A., et al. (2019) 2018 ESC/EACTS Guidelines on Myocardial Revascularization. European Heart Journal, 40, 87-165. 